\title{
Depletion interaction mediated by polydisperse rods
}

\author{
Peter R. Lang* \\ Forschugszentrum Jülich, Institut für Festkörperforschung, \\ Weiche Materie, D-52425 Jülich, Germany
}

\begin{abstract}
The interaction between a colloidal hard sphere of radius $R$ and a wall or between two spheres in a dilute suspension of infinitely thin rods of length $L$ is calculated numerically. The method allows to study depletion potentials for any value of $L / R$ and consequently the influence of rod length polydispersity can be investigated. It was observed that both the depth and the range of the potential increase drastically if the relative standard deviation, $\sigma$, of the length distribution is larger than 0.25 , while the potential is virtually indistinguishable from that caused by monodisperse rods, if $\sigma \lesssim 0.1$
\end{abstract}

\footnotetext{
* email: p.lang@fz-juelich.de
} 


\section{Introduction}

Depletion interactions occur when two or more kinds of colloidal particles of different size or colloidal particles and polymers are mixed in a suspending medium. If two particles of one species approach each to such a degree that the other species is excluded from the gap between them, the resulting osmotic pressure imbalance causes an attractive force between the two particles. Asakura and Oosawa [1] were the first to calculate the depletion force between two plates immersed in a dilute solution of ideal polymer chains. In the same contribution they considered the case two spherical bodies in a solution of small spheres. Since then a wealth of theoretical and experimental work especially on depletion interaction between colloids mediated by polymer chains or a second globular species has been published (for an overview see the reviews by Poon [2] and Tuinier et al. [3]).

Asakura and Oosawa were again the first to calculate the attractive force between two parallel plates in a solution of infinitely thin rigid rods [4]. Auvray derived a closed analytical expression for the potential between two spheres in a dilute suspension of rods with $L / R \ll$ 1 [5]. Later Mao et al published [6] a series expansion in density up to third order for the depletion potential mediated by rods of finite aspect ratios in the Derjaguin limit. Although rod-like particles are expected to be much more effective depletants than spherical particles or polymers, there have been only a few experimental studies in which depletion potentials were measured directly. Yodh and coworkers have thoroughly studied the depletion potential induced by bacteriophage fd-virus $[7,8]$ by optical tweezer experiments. The potential between flat glass wall and a latex bead in solutions of boehmite rods was measured with Total Internal Reflection Microscopy (TIRM) by Helden et al [9, 10].

Surprisingly, the effect of a finite size distribution of the depletant on the interaction has attracted limited attention. Most of the theoretical and computer modelling work on the 
effect of polydispersity was done on polydisperse spheres [11-16] as depletant. Tuinier and Petukhov calculated the depletion force between two plates in a solution of polydisperse ideal chain macromolecules [17]. For the case of rod-like depletants no systematic study on polydispersity effects has been published so far although Yaman et al. published a numerical method $[18,19]$ to calculate the depletion interaction between two spheres of radius $R$ mediated by an ideal gas of infinitely thin rods of length $L$ for arbitrary $L / R$.

To our knowledge there are only a few contributions, in which the effect of polydispersity has been studied systematically in experiments. Piech et al. found semi quantitative agreement between theoretical predictions and their atomic force microcopy measurements of the depletion potential between a spherical silica particle and a silica flat mediated by an ensemble of polydisperse nanospheres [20]. Kleshchanok et al. measured interaction profiles between a colloidal sphere and a wall caused by polydisperse macromolecules with TIRM [21]. It was shown that the experimental data can not be described correctly using averaged values of the polymer size distribution. If the width of the distribution is large, rather the full functional form of the size distribution has to be accounted for. The depletion interaction between a wall and a sphere due to boehemite rods was investigated by Helden et al. $[9,10]$. However, in this case the influence of polydispersity was not considered explicitly.

In this paper we will report on the numerical calculation of the the depletion potential between a sphere and a wall or two spheres mediated by an ideal gas of rods in the limit of small rod number density. The numerical integrations were done along the line described by Yaman et al. for the interaction between two spherical colloids in a rod solution. Since this algorithm yields exact values for the potential within numerical accuracy for any $L / R$ it can be extended to the calculation of the potential mediated by an ensemble of polydisperse rods. In the method section we give a brief outline of the calculation scheme before. Subsequently, 
the effect of polydispersity for a range of radius to mean rod length ratios is discussed for the sphere/wall and the sphere/sphere case in the results and discussion section. Finally, the details of the numerical integrations are presented in an appendix.

\section{Method}

The depletion interaction between two rigid bodies mediated by an ideal gas of rigid infinitely thin rods can be visualized as the free energy change caused by the immersion of the two bodies at infinite distance and subsequently bringing them to their final separation distance $D$. If the rods are treated as an ideal gas and the interaction with the two bodies is only due to excluded volume the resulting potential between the two bodies is given by

$$
\phi(D)=\Pi\left[V_{e x}(D)-V_{e x}(\infty)\right]
$$

Here $V_{e x}(\infty)$ is the volume excluded for the rods by the two rigid bodies at infinite separation. For the case of two spheres $V_{e x}(\infty)=2 V_{e x}^{S}$ while for the case of a wall and a sphere $V_{e x}(\infty)=$ $V_{e x}^{W}+V_{e x}^{S}$. Further on the superscript $S$ will denote quantities related to a spherical particle, while $W$ refers to a wall. The volume excluded for the rods by a single wall or a single sphere is given by

$$
\begin{aligned}
V_{e x}^{W} & =\frac{L}{4} \times A \\
V_{e x}^{S} & =\frac{4 \pi}{3} R^{3}+\int_{R}^{R+L / 2} d K K^{2}(1-\cos \delta(K, R))
\end{aligned}
$$

respectively, where $A$ is the wall area. As illustrated in Figure $1, K$ is the sphererod center to center distance and $\delta$ the angle included between $K$ and the rod. Thus $\cos \delta(K, R)=\sqrt{K^{2}-R^{2}} / K$ if $K<\sqrt{R^{2}+(L / 2)^{2}}, \cos \delta(K, R)=\left(K^{2}+(L / 2)^{2}-R^{2}\right) /(K L)$ if $\sqrt{R^{2}+(L / 2)^{2}} \leq R+L / 2$ and $\cos \delta(K, R)=0$ elsewhere. The total excluded volume at 
finite separation distance between a wall and a sphere is most generally given by

$$
V_{e x}(D)=\int d \mathbf{r} \frac{1}{4 \pi} \int d \bar{\omega} c(\mathbf{r}, \bar{\omega})
$$

where the Mayer-like characteristic function $c(\mathbf{r}, \bar{\omega})=1-\exp (1-u(\mathbf{r}, \bar{\omega}))$ is unity if the rod touches either the sphere or the wall and $c(\mathbf{r}, \bar{\omega})=0$ otherwise, if $u(\mathbf{r}, \bar{\omega})$ is the excluded volume interaction of the rod with the two bodies. The first integral in eq. 4 represents the integration over all center positions of the rod and the second serves to calculate the fraction of all possible orientations of the rod, which are not accessible for a given center position. Thus, eq. 4 in principle represents a fivefold integration, which however can be reduced to a twofold ore three fold integration depending on the succession of the angles $\delta_{s l}$, $\delta_{w l}, \delta_{w u}$ and $\delta_{s u}$ as is described in detail in the Appendix. For the case of monodisperse rods with arbitrary length, these integrations can be performed in a few minutes on a personal computer. The same algorithm can be used to calculate $V_{e x}(D)$ for a sphere/wall as well as for a sphere/sphere configuration, if the wall in Fig. 1 is replaced by a second sphere such that the angles $\delta_{w l}$ and $\delta_{w u}$ remain unchanged. This is indicated by the dotted sphere in Fig. 1.

The osmotic pressure for an ideal gas of rods is given by $\Pi=\rho k_{B} T$ where $\rho$ is the rod number density, with which the depletion potential can be written in thermal units as

$$
\frac{\phi(D)}{k_{B} T \rho}=V_{e x}(D)-V_{e x}(\infty)
$$

The depletion interaction mediated by a polydisperse ensemble of rods can now be calculated from

$$
\frac{\phi_{p}(D)}{k_{B} T \rho}=\frac{\int_{0}^{\infty} d L \Psi(L)\left[V_{e x}(D)-V_{e x}(\infty)\right]}{\int_{0}^{\infty} d L \Psi(L)}
$$

where $\Psi(L)$ is the distribution function of the rod lengths, for which we choose a logarithmic 


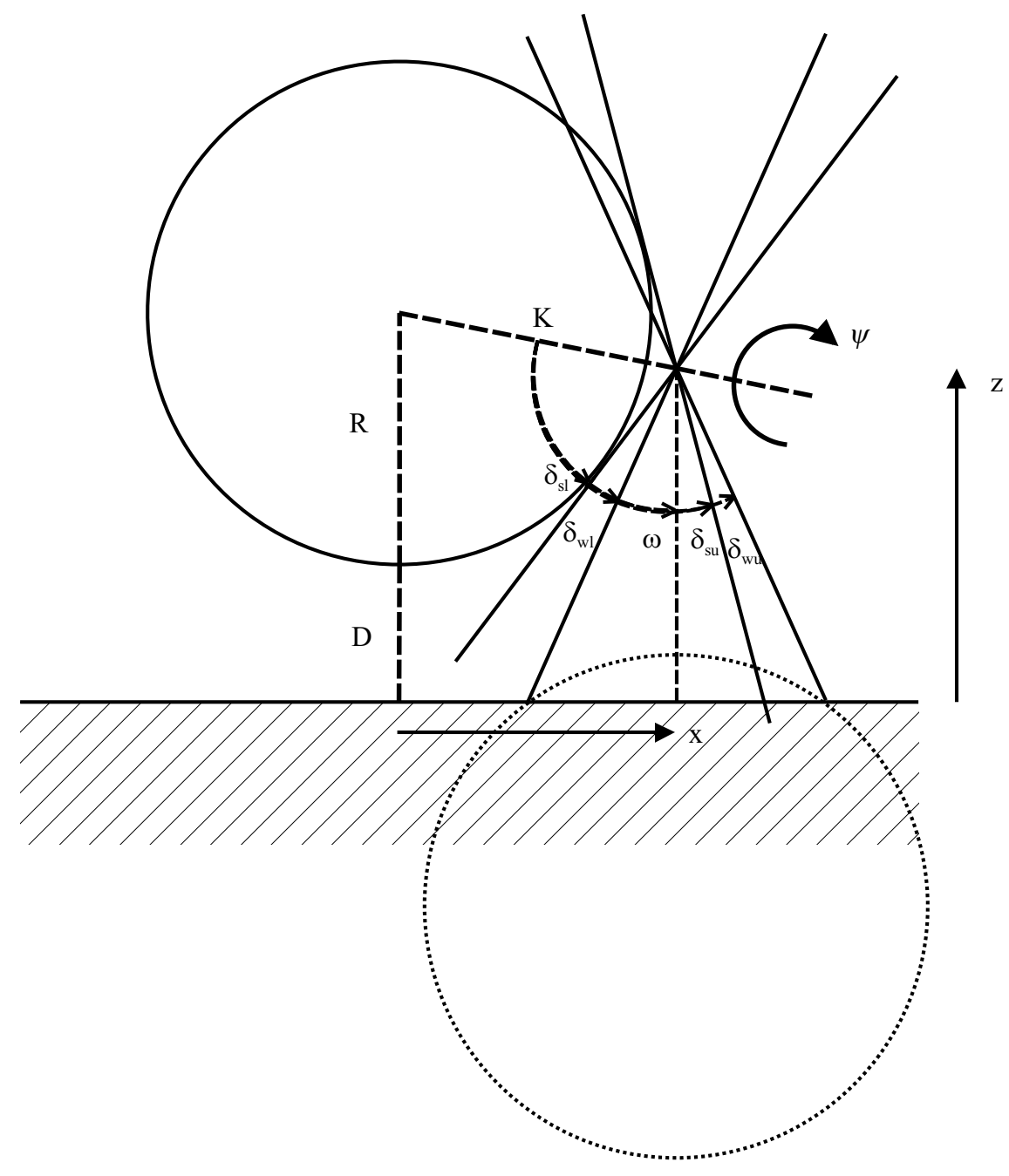

FIG. 1: Basic construction for the calculation of the volume excluded, $V_{e x}(D)$, for the rod by a sphere and a wall. $V_{e x}(D)$ is calculated by numerical integration as a function of the rod's center position and its orientation. The dotted sphere excludes the same volume for the rod as the wall. With this construction it is also possible to calculate $V_{e x}(D)$ from two spheres. normal distribution

$$
\Psi(L)=\frac{1}{L \sigma \sqrt{2 \pi}} \exp \left\{-\frac{[\ln (L /<L>)]^{2}}{2 \sigma^{2}}\right\}
$$

Here $\langle L>$ is the average length and $\sigma=\Delta L /<L>$ the relative standard deviation, with $\Delta L$ the standard deviation. 
The numerical accuracy of the integration was checked by stepwise increasing the number of pivot points until a further increase by a factor of two changed the computed values by less than 0.1 percent. Accordingly, 500 pivot points were necessary for the volume and the orientational integration, if $L / R<<1$ while 100 pivot point were sufficient in the opposite case. Since the length distribution function is rather well behaved 10 pivot points were more than sufficient in any case. Further, the algorithm was tested against two known limiting cases. The potential mediated by monodisperse rods at low density is conveniently written as

$$
\frac{\phi(D)}{k_{B} T}=\rho L^{2} R K_{1}(D)
$$

where $K_{1}(D)=-\pi(1-D / L)^{3} / 6$ is a good approximation for the sphere/sphere configuration $[5,6]$ in the Derjaguin limit, i. e. if $L / R<<1$. For the sphere/wall configuration in the same limit $K_{1}(D)=-\pi(1-D / L)^{3} / 3$. These limits are reproduced within numerical accuracy by our calculations, as is shown for both cases in Fig. 2

As a second test we reproduced the data published by Yaman et al. [19] for $K_{1}(D=0)$ as a function of $L / R$ for the sphere/sphere configuration, which had been fitted to the function

$$
K_{1}(D=0)=-\frac{\pi}{6} \frac{1+A(L / R)}{1+B(L / R)+C(L / R)^{2}} .
$$

In the range $0.05<L / R<13$ Yaman et al. found $A=0.8762, B=1.33198$ and $C=0.9825$. In Fig. 3 we compare our numerical data to this fit and find good agreement in the complete range of $L / R$.

\section{Results and Discussion}

Monodisperse rods of arbitrary length

The depletion potential between two colloidal spheres mediated by monodisperse rods has 


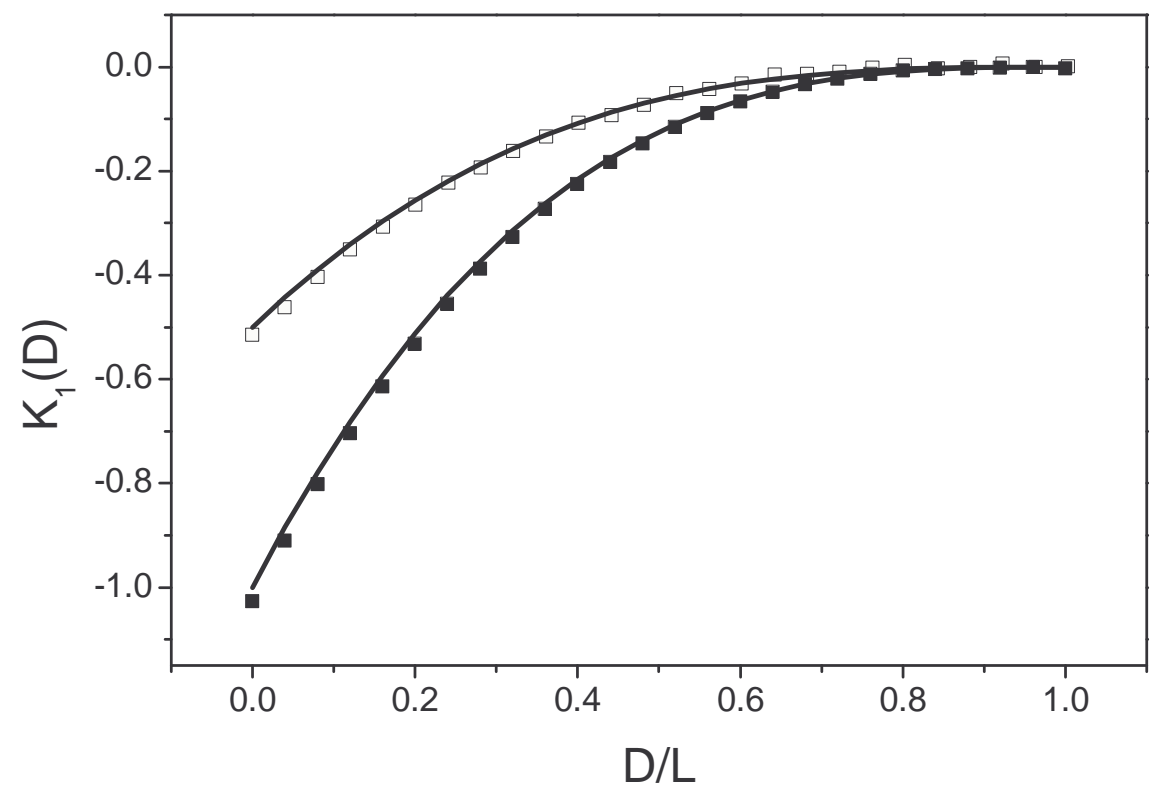

FIG. 2: Comparison of numerically exact results for $K_{1}(D)$ as a function of $D / L$ with the prediction from Derjaguin's approximation. Symbols are data obtained from numerical calculation of the excluded volume in the sphere/sphere (open) and the sphere/wall (solid) configuration for $L / R=$ 0.1. Full lines represent the corresponding Derjaguin approximation.

been calculated by Yaman et al. for a wide range of $L / R$ [19]. In Fig. 4 we present the according data for the sphere/wall configuration, as $K_{1}(D)$ vs. $D / L$. Similar to the earlier results we find that the Derjaguin approximation is very good for $L / R \leq 0.5$ and even at $L / R=1$ the approximation deviates from the exact results significantly only at separation distances smaller than $0.2 L$. Further, it appears that the functional form of the distance dependence of the approximation can give a rough estimate of the potential shape up to $L / R=10$. This is shown by the dotted lines in Fig. 4, which represent fits to the calculated data by $K_{1}(D)=-\alpha(1-D / L)^{3}$, with $\alpha$ being the fit parameter. The contact values of $K_{1}$ are plotted in Fig. 3 which also follow the Derjaguin approximation, i. e. $K_{1}(D=0)=-\pi / 3$ for $L / R \lesssim 0.5$. The numerical data can be fitted by eq. 9 keeping $A$ and $B$ fixed to the values given by Yaman et al. and allowing for a factor of two in the pre-factor. The result 


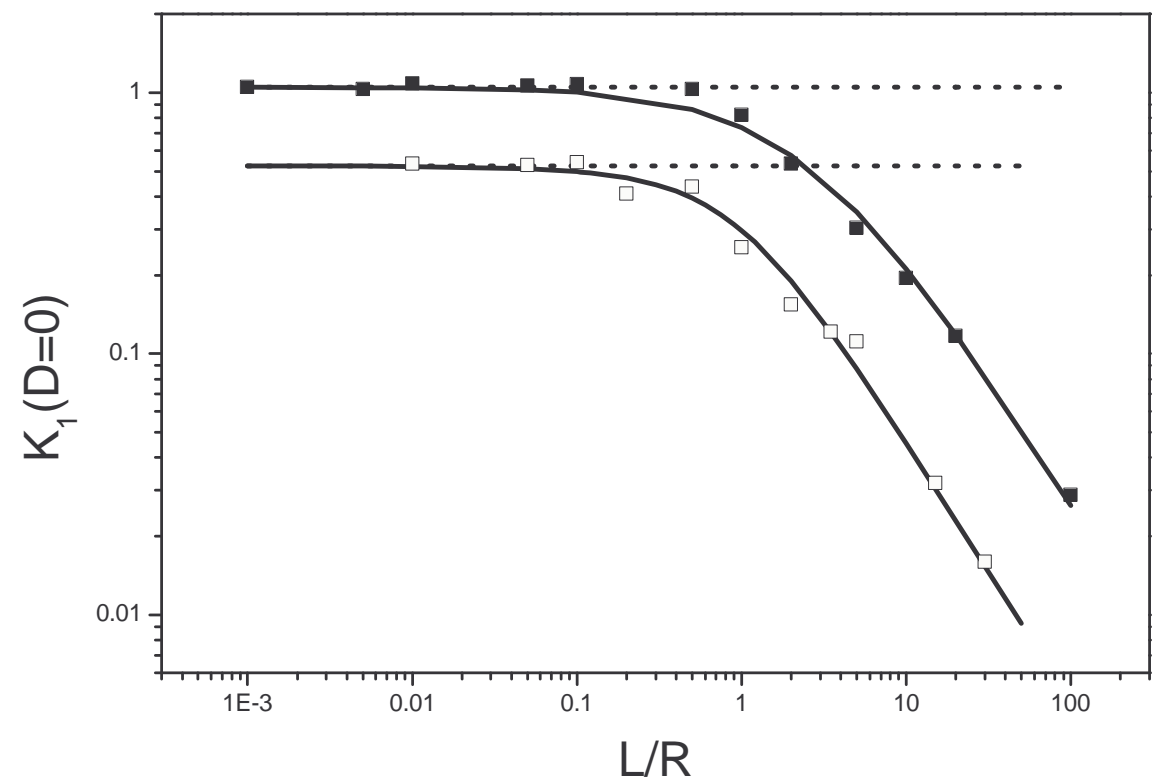

FIG. 3: Dependence of $K_{1}(D=0)$ on $L / R$. Symbols are data obtained from numerical calculations for the sphere/sphere (open) and the sphere/wall (solid) configuration. Full lines represent three parameter fits with eq. 9 and the dotted horizontal line are the predictions by the Derjaguin approximation.

for $C=0.341$ is shown as solid curve in the figure.

The small but significant upturn of the numerical data at small distances was not obvious from the distance dependence of $K_{1}(D)$ published by Yaman et al., for the sphere/sphere case. However, Lau et al. published experimental data for the sphere/sphere interaction mediated by the rod-like fd-virus [8] and compared these to numerical results, which were calculated with Yaman's algorithm. In this paper, both the experimental and the numerical data show a pronounced upturn at small separation distances. Further, we also find this upturn in our numerical results for the sphere/sphere geometry, as is also shown in Fig. 4. Our $K_{1}(D=0)$ values, which for the sphere/sphere configuration match very well with the three-parameter fit by Yaman et al. [19] were extracted from potential curves showing this upturn. We conjecture that the upturn did not display in the original paper due to 


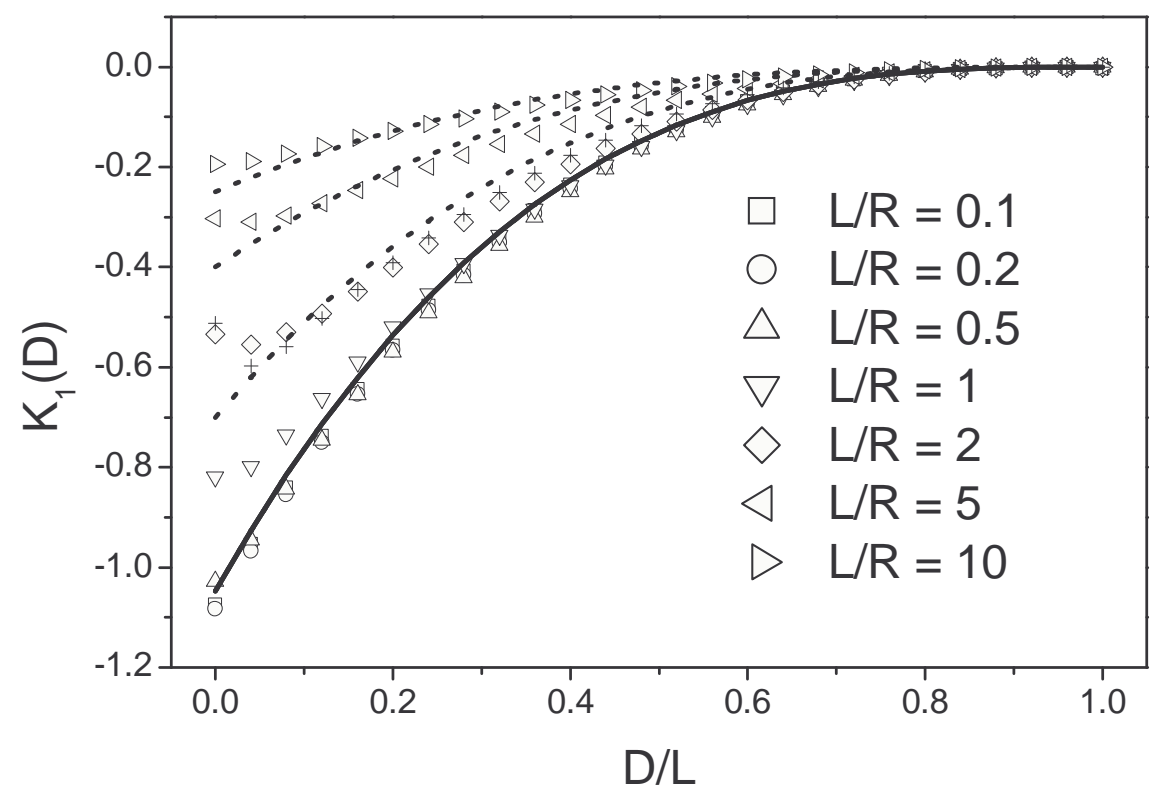

FIG. 4: Dependence of $K_{1}(D)$ on $D / L$ in the sphere/wall configuration for different $L / R$ as indicated in the legend. The crosses represent the twofold of the potential calculated for $L / R=1$ in the sphere/sphere configuration. The full line represents the Derjaguin approximation and the dotted curves are a rough guide assuming the same $D / L$ dependence with an amplitude which changes with $L / R$.

the reduced $D / L$-resolution. Therefore we are convinced this upturn is not a numerical artifact, although it is counterintuitive, because a repulsive contribution to the interaction potential requires an increase of the total excluded volume, $V_{\text {ex }}(D)$, with decreasing $D$ according to eq. 5. A qualitative geometrical explanation for this observation is sketched in Fig. 5. The excluded volume at finite distance, $V_{e x}(D)$, is composite of two counter balancing contributions. On approach of two spheres the overlap of the depletion zones yields additional free volume in the system, which is accessible to the center of mass of rods, such that no restriction of their orientation occurs by either of the two spheres. This so called overlap volume increases on further approach of the spheres, leading to a decrease of $V_{e x}(D)$. 

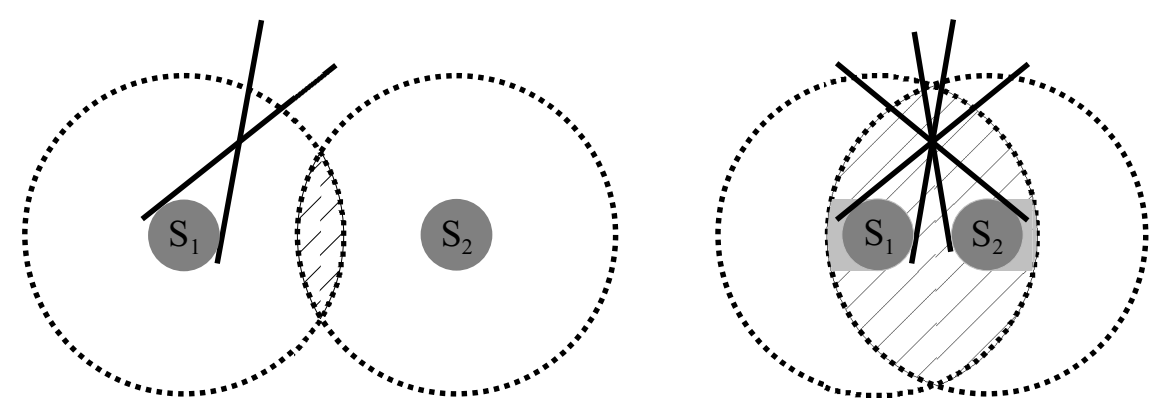

FIG. 5: Illustration of the counter balancing effects of positional and orientational degrees of freedom. Left: the depletion zones of two spheres indicated by the dotted circles overlap, while the orientation of the rod is hindered only by sphere $S_{1}$. Right: The overlap volume, which is related to the hatched area, increases on further approach of the spheres, leading to a decrease of $V_{e x}(D)$. On the other side, the rod experiences an additional restriction of orientational freedom due to sphere $S_{2}$, which increases $V_{e x}(D)$.

However, for the rod sketched in Fig. 5, the accessible range of orientations decreases at the same time, because it is restricted by two spheres now. The same holds for all rods, which have their center of mass position in the overlapping depletion zones, except for those in the 'shadow' of either sphere, the area which is indicated in light grey in Fig. 5. This additional restriction of orientation leads to a significant increase of $V_{e x}(D)$, which might for certain combinations of $R, L$ and $D$ overcompensate the gain of overlap volume.

\section{Polydisperse rods}

The dependence of the depletion potential on polydispersity is shown in Fig. 6 for $<L>$ $/ R=1$. For both the the sphere/sphere and the sphere/wall configuration no deviation from the potential mediated by monodisperse rods can be observed if $\sigma \lesssim 0.1$. Even for a relative standard deviation of $25 \%$ the difference is smaller than would be discernible 
with state of the art experimental techniques like TIRM [22] or optical tweezing (OT) [23] techniques. At larger standard deviations, both the absolute contact value and the range of the potential increase with $\sigma$. This effect is more pronounced for the sphere/wall than for the sphere/sphere configuration. The threshold separation distance, $d_{0.1} \equiv(D /<L>)_{0.1}$, at which the absolute potential value has dropped below $10 \%$ of the absolute contact value, is always larger at constant $\sigma$ for the sphere/wall configuration, e. g. for the sphere/wall data displayed in Fig. $6 d_{0.1}=1.2$ at $\sigma=0.5$, while $d_{0.1}=0.9$ for the sphere/sphere configuration. At $\sigma=0.75$ the corresponding values are $d_{0.1}=2.0$ and $d_{0.1}=1.4$ respectively.

The depth of the interaction potential also strongly increases with the relative standard deviation of the rod length distribution. In Fig. 7 we present the contact values normalized to the contact value for monodisperse rods as a function of $\sigma$ for a wide range of $\langle L>$ $/ R$ values. Also for this quantity the effect of polydispersity is more pronounced for the sphere/wall configuration, although the difference to the sphere/sphere configuration is not as evident as for the potential range. The contact potential depends most strongly on $\sigma$ if $<L>/ R<<1$. In this case a standard deviation of $75 \%$ will increase the absolute contact value by a factor of about 35 with respect to the case of monodisperse rods. But even in the opposite limit where the potential is very weak anyway, it is deepened by a factor of about $10-15$ at $\sigma=0.75$.

The observed increase of the absolute contact value with $\sigma$, at first glance appears to be in contradiction to the results of Mao who predicted that polydispersity greatly reduces the magnitude of the depletion force between two large spheres in a solution of small polydisperse spheres [11]. Further, the hight of the repulsive maximum of the potential profile, which is expected at high depletant content is reduced by polydispersity. Piech and Walz reported a similar effect for the potential between two charged spheres in a solution of polydisperse 


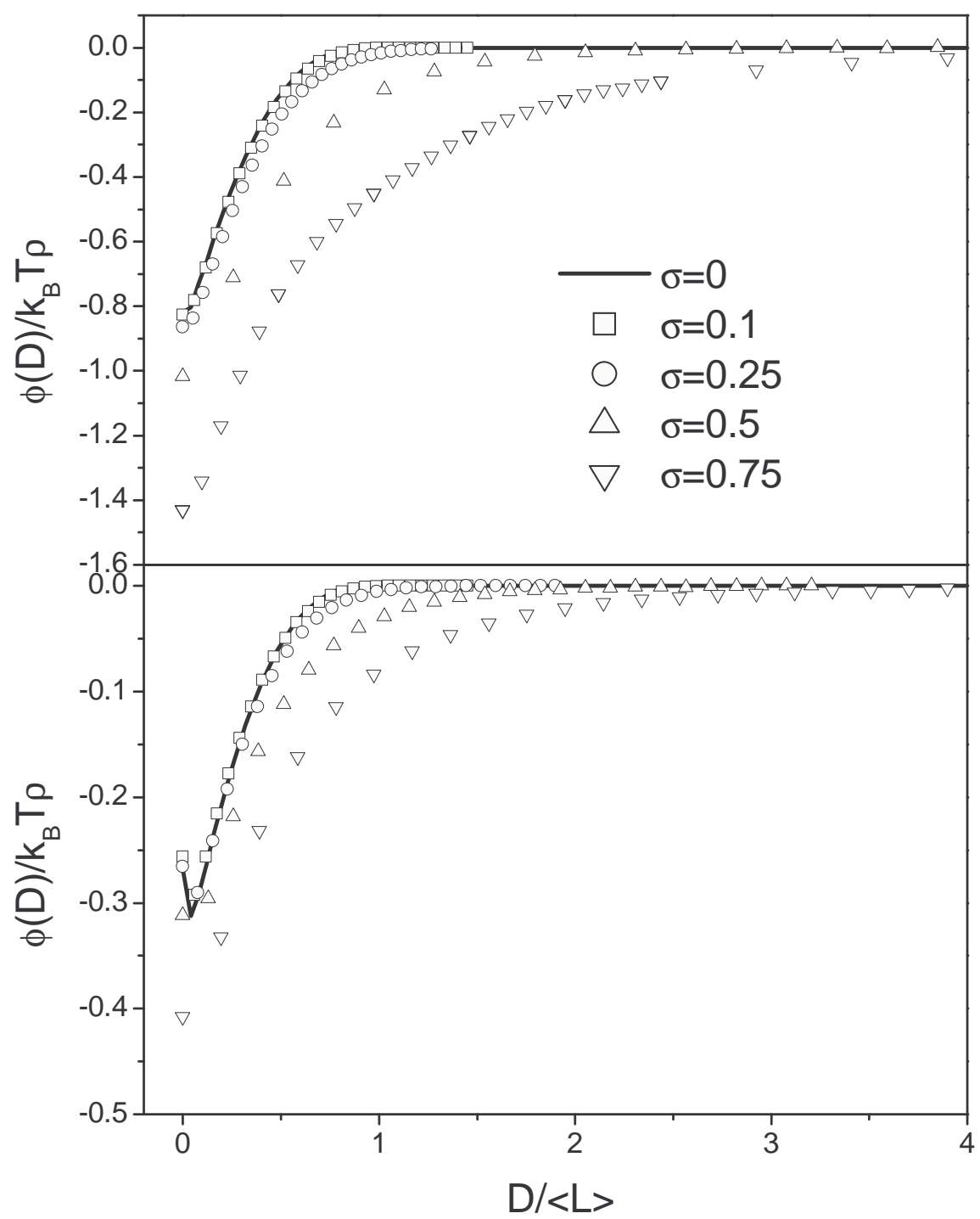

FIG. 6: Depletion potentials mediated by polydisperse rods with $\langle L\rangle / R=1$ vs. normalized separation distance for sphere/wall (top) and sphere/sphere (bottom) configuration. Symbols refer to different values of the standard deviation as indicated in the legend. The full lines represent the case of monodisperse rods.

charged small spheres [15]. However, it is important to note that these calculations were performed for constant volume fraction of the depleted spheres, in which case, the number density of the depletants changes with polydispersity. If the same calculations are performed at constant number density both the absolute contact value and the height of the repulsive 
maximum increase with $\sigma$. Concerning the dependence of the potential contact value on polydispersity, our results are therefore well inline with these earlier calculations, although it has to emphasized that we are neglecting the effect of rod-rod interaction. Therefore this approach will not predict the repulsive maximum which is also expected for rods at higher densities [6].

It is further interesting to investigate how the depletion potentials vary with the exact shape of the rod length distribution. For this purpose we compare the calculated potential between two spheres mediated by rods with a log-normal (LN) distribution and a Schulz-Zimm (SZ) distribution in Fig. 8. The displayed data were calculated for $\langle L\rangle / R=1$, however the general trend is the same for all $\langle L\rangle / R$ investigated. The major differences between the potentials calculated with different distribution functions are a larger absolute contact value and a shorter range of the potentials mediated by SZ distributed rods. This can be qualitatively understood from the shape of the distributions, which are displayed in the inset of Fig. 8. In the SZ distribution the short rods have a higher frequency than in the LN distribution, which leads to a deeper interaction potential at small separation distances and a reduced range. However, these effects are not very pronounced, for example the difference of the contact potential is less than $30 \%$ even at $\sigma=0.75$.

So far all potentials were compared at constant number density of the rods. For this situation we observed that increasing polydispersity of the rods will lead to an increase of the absolute contact value and of the potential range. However, in technical applications, e. g. flocculation or induce phase transitions of of colloidal dispersions by the addition of rods, and for the experimental determination of interaction potentials, usually the mass concentration $c$ of the rods is preset. Therefore, it is important to note that the number 


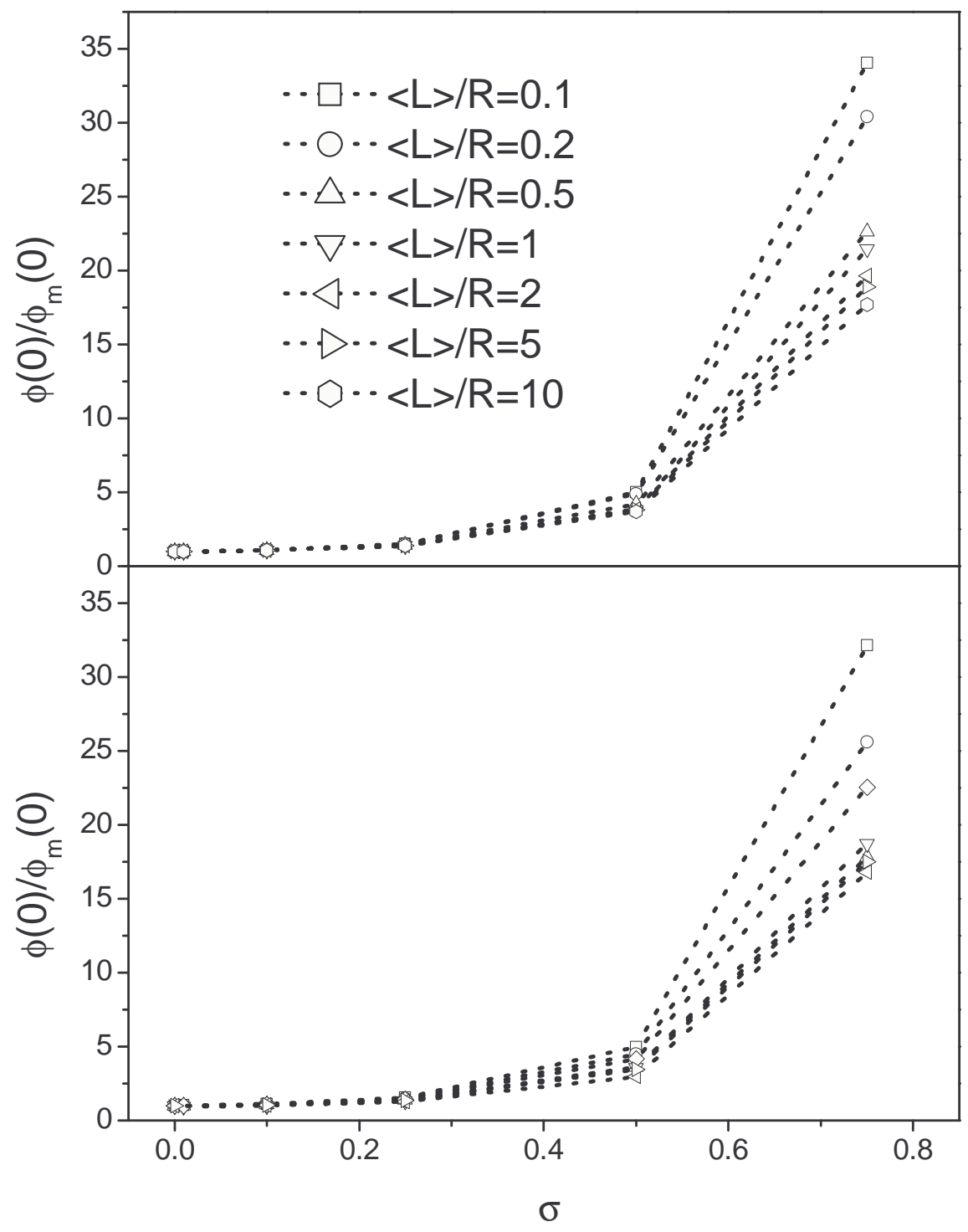

FIG. 7: Contact potentials normalized to the value for monodisperse rods as a function of relative standard deviation for sphere/wall (top) and sphere/sphere (bottom) configuration. Symbols are calculated for different $\langle L\rangle / R$ as indicated in the legend and dotted lines are guides to the eye.

density varies with the width of the distribution

$$
\rho=\frac{c}{\rho_{L}} \frac{\int_{0}^{\infty} \frac{\Psi(L)}{L} d L}{\int_{0}^{\infty} \Psi(L) d L},
$$

if the concentration is fixed as , where $\rho_{L}$ is the mass per unit length of the rods. For 


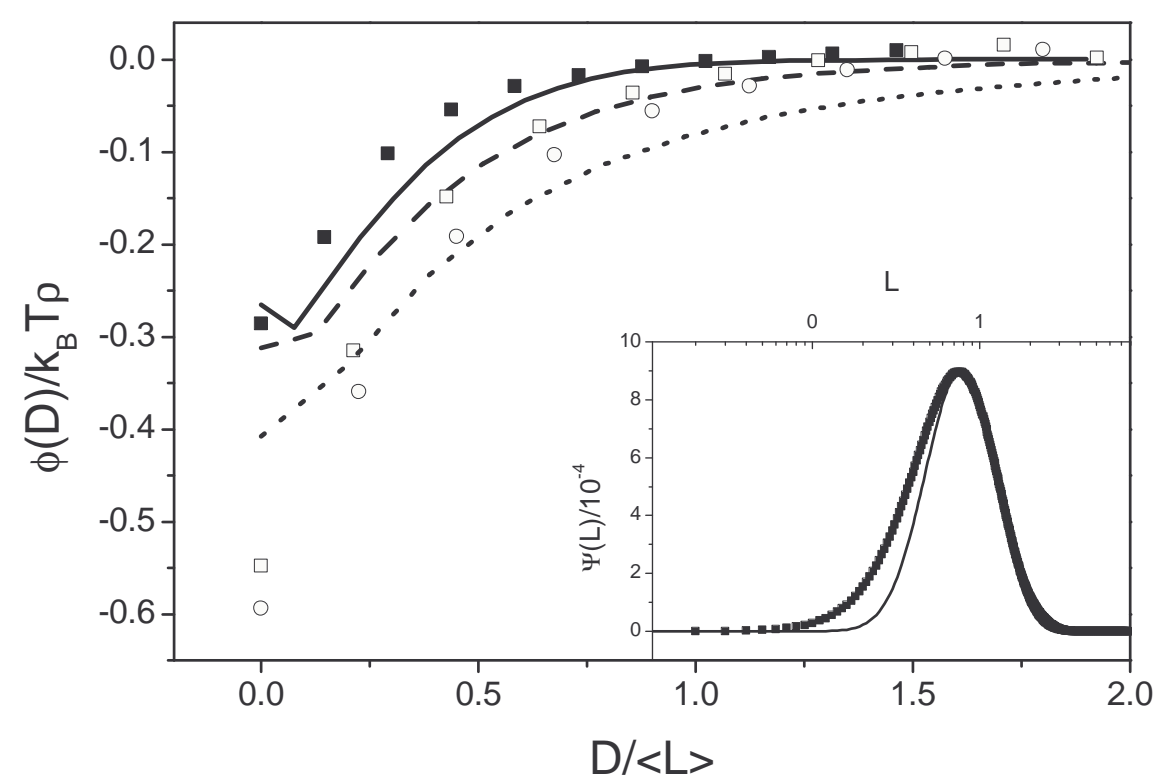

FIG. 8: Depletion potentials mediated by polydisperse rods. The lines represent potentials mediated by $\log -$ norm distributed $(\sigma=0.25$ : full, $\sigma=0.5$ : dotted and $\sigma=0.75$ : dashed) ensembles of rods, while the symbols correspond to Schulz-Zimm distributions with $\sigma=0.25$ : full squares, $\sigma=0.5$ : open squares and $\sigma=0.75$ : open circles. Inset: Log-norm (full line) and Schulz-Zimm (symbols) distribution function with $\sigma=0.5$

monodisperse rods eq. 10 gives $\rho=c /\left(L \rho_{L}\right)$, while for a log-normal distribution it is as weakly increasing function of $\sigma$. However, this effect is only significant for relative distribution widths $\sigma \geq 0.5$, as is shown in Fig. 9. There we show as an example two sets of potentials which calculated for $\langle L>/ R=0.1$. In the first set the number density was fixed to $\rho=955 \mu \mathrm{m}^{-3}$ and in the second the mass concentration and the mass per unit length were chosen such to give $c / \rho_{L}=955 \mu \mathrm{m}^{-3}$.

\section{Conclusions}

We have calculated the effect of length polydispersity on the depletion potential mediated by a dilute suspension of infinitely thin rods between a sphere and a wall as well as between two 


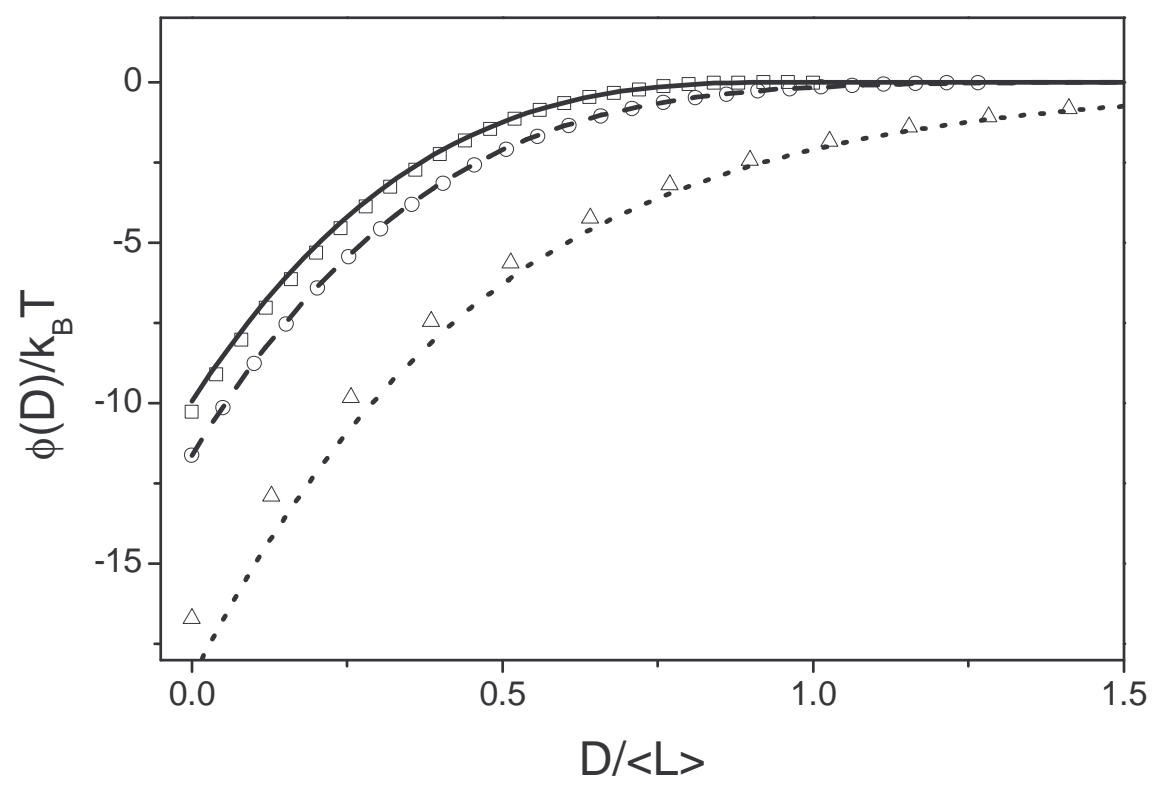

FIG. 9: Depletion potentials mediated by an ensemble of rods with varying width of the length lognormal distribution. The symbols are exact potentials calculated for constant rod number density, $\rho=955 \mu \mathrm{m}^{-3}$, with $\sigma=0$ (squares), $\sigma=0.25$ (circles) and $\sigma=0.5$ (triangles). The lines represent Derjaguin approximations at constant mass concentration, $c=7.455 \mathrm{~g} / \mathrm{L}$ with with $\sigma=0$ (full), $\sigma=0.25$ (dotted) and $\sigma=0.5$ (dashed).

spheres of equal radius. Since higher order density effects arising from interactions between the rods themselves are neglected, the model will not predict repulsive contributions to the depletion potential and oscillatory structural forces. For relative standard deviations of the length distribution smaller than 0.25 the effect would be hardly discernible by modern experimental techniques. However with increasing $\sigma$ at constant rod number density, length polydispersity leads to an increase of the potential depth and range, in accordance with earlier predictions for spherical depletants. As a general trend we observed that this effect is more pronounced for the sphere/wall configuration than for the sphere/sphere case. Further, we observed that the exact shape of the distribution function is of minor importance. Finally, it is noteworthy for technical applications that polydisperse rods are more efficient depletants 
than monodisperse rods at the same mass concentration.

\section{Acknowledgement}

This work did benefit substantially from discussions with Remco Tuinier and Gerrit Vliegenthart, which I am really grateful for.

\section{Appendix}

The expression for the excluded volume of eq. 4 is most conveniently written in cylindrical coordinates for the rod center position as

$$
V_{e x}(D)=2 \pi \int_{0}^{R+L / 2} x d x \int_{0}^{D+2 R+L / 2} d z I_{o}
$$

with

$$
I_{o}=\frac{1}{4 \pi} \int \sin \delta d \delta \int d \psi(1-\exp \{-u(x, z, \psi, \delta)\}
$$

where the factor $2 \pi$ stems from the integration over the angular coordinate, and the upper limits of the $x$ and $z$-integration are the maximum value for either coordinate, at which the rod can touch the sphere. The latter is located with its center at $x=0$ and $z=D+R$. The orientation of the rod is defined by the inclination angle, $\delta$, between the center to center connection and the rod and by the azimuthal angle, $\psi$, around the center to center connection. We have now to consider three different situations.

(i) If the center position of rod is such that the rod can not touch the wall, i. e. $z>L / 2$ then the characteristic function $c(\mathbf{r}, \delta, \psi)=1$ for $0 \leq \psi \leq 2 \pi$ if $\delta_{s l} \leq \delta \leq \delta_{s u}$. Accordingly the orientational integration $I_{o}$ gives

$$
I_{o}=1-\cos \delta_{s l}
$$

since $\delta_{s u}=\pi-\delta_{s l}$. According to Fig. $1 \cos \delta_{s l}=\sqrt{K^{2}-R^{2}} / K$ if $R<K \leq \sqrt{R^{2}+(L / 2)^{2}}$, 
$\cos \delta_{s l}=\left(K^{2}+(L / 2)^{2}-R^{2}\right) /(K+L)$ in the range $\sqrt{R^{2}+(L / 2)^{2}}<K \leq R+L / 2$ and $\cos \delta_{s l}=1$ elsewhere. If the center of the rod is located in the volume of the sphere $I_{o}=1$.

(ii) If the free rotation of the rod is hindered only by the wall, the orientational integration is given by

$$
I_{o}=\frac{1}{4 \pi} \int_{\delta_{w l}}^{\delta_{w u}} \sin \delta d \delta \int_{-\psi_{l}}^{\psi_{l}} d \psi
$$

where the integration limits for the azimuthal angle depend on the angle between the center to center line and the wall normal $\omega$, i. e. the position of the sphere. If the sphere is located entirely outside the conical volume excluded by the wall

$$
\psi_{l}=\cos ^{-1}\left[\frac{2 z / L-\cos \omega \cos \delta}{\sin \omega \sin \delta}\right]
$$

On the other hand, if the sphere is entirely located within the cone then $\psi_{l}=\pi$ in the ranges $\delta_{w l} \leq \delta \leq \omega$ and $\omega \leq \delta \leq \delta_{w u}$ while eq. 15 applies elsewhere. Note that in both cases the result of the integration has to be

$$
I_{o}=1-\cos \left(\omega-\delta_{w l}\right)
$$

which is obtained if the azimuthal rotation is performed around the surface normal.

(iii) If the center of the rod is located such that the rod may touch both the sphere and the wall upon variation of $\delta$, appropriate combinations of eqs. 13 and 14 have to be applied, depending on the succession of the angles $\delta_{s l}, \delta_{s u}, \delta_{w l}$ and $\delta_{w u}$. For example, in the situation depicted in Fig. 1 the orientational integration gives

$$
I_{o}=1-\cos \delta_{s l}+\frac{1}{4 \pi} \int_{\delta_{w l}}^{\delta_{s u}} \sin \delta d \delta 2 \cos ^{-1}\left[\frac{2 z / L-\cos \omega \cos \delta}{\sin \omega \sin \delta}\right]
$$

for $\delta_{s l}<\delta_{w l}<\delta_{s u}<\delta_{w u}$ 


\section{References}

[1] S. Asakura and F. Oosawa J. Chem. Phys. 22, 1255 (1954).

[2] W. C. K. Poon Curr. Opin. Colloid Interface Sci. 3, 593 (1998).

[3] R. Tuinier, J. Rieger, and C. G. de Kruif, Adv. Colloid Interface Sci 103, 1 (2003).

[4] S. Asakura and F. Oosawa J. Polym. Sci. 33, 183 (1958).

[5] L. Auvray, J. Phys. (Paris) 42, 79 (1981).

[6] Y. Mao, M. E. Cates, and H. N. W. Lekkerkerker, J. Chem. Phys. 106, 3721 (1997).

[7] K.-H. Lin, J. C. Crocker, A. C. Zeri, and A. G. Yodh Phys. Rev. Lett. 87, 088301 (2001).

[8] A. W. C. Lau, K.-H. Lin, and A. G. Yodh, Phys. Rev. E 66, 020401(R) (2002).

[9] L. Helden, R. Roth, G. H. Koenderink, P. Leiderer, and C. Bechinger, Phys. Rev. Lett. 90, 048301 (2003).

[10] L. Helden, G. H. Koenderink, P. Leiderer, and C. Bechinger, Langmuir 20, 5662 (2004).

[11] Y. Mao, J. Phys. II 5, 1761 (1995).

[12] Y. Walz, J. Colloid Interface Sci. 178, 505 (1996).

[13] P. B. Warren, Langmuir 13, 4388 (1997).

[14] R. P. Sear and D. Frenkel, Phys. Rev. E 55, 1677 (1997).

[15] M. Piech and J. Y. Walz, J. Colloid Interface Sci. 225, 134 (2000).

[16] D. Goulding and J. P. Hansen, Mol. Physs 99, 865 (2001).

[17] R. Tuinier and A. V. Pethukhov, Macromol. Theory Sim. 11, 975 (2002).

[18] K. Yaman, M. Jeng, P. Pincus, C. Jeppesen, and C. M. Marques, Physica A 247, 159 (1997).

[19] K. Yaman, C. Jeppesen, and C. M. Marques, Europhys. Lett. 42, 221 (1998). 
[20] M. Piech and J. Y. Walz, J. Colloid Interface Sci. 253, 117 (2002).

[21] D. Kleshchanok, R. Tuinier, and P. R. Lang, Langmuir 22, 9121 (2006).

[22] D. C. Prieve, Adv. Colloid Interface Sci. 82, 93 (1999).

[23] D. G. Grier, Curr. Opin. Coll. Interf. Sci. 2, 264 (1997). 


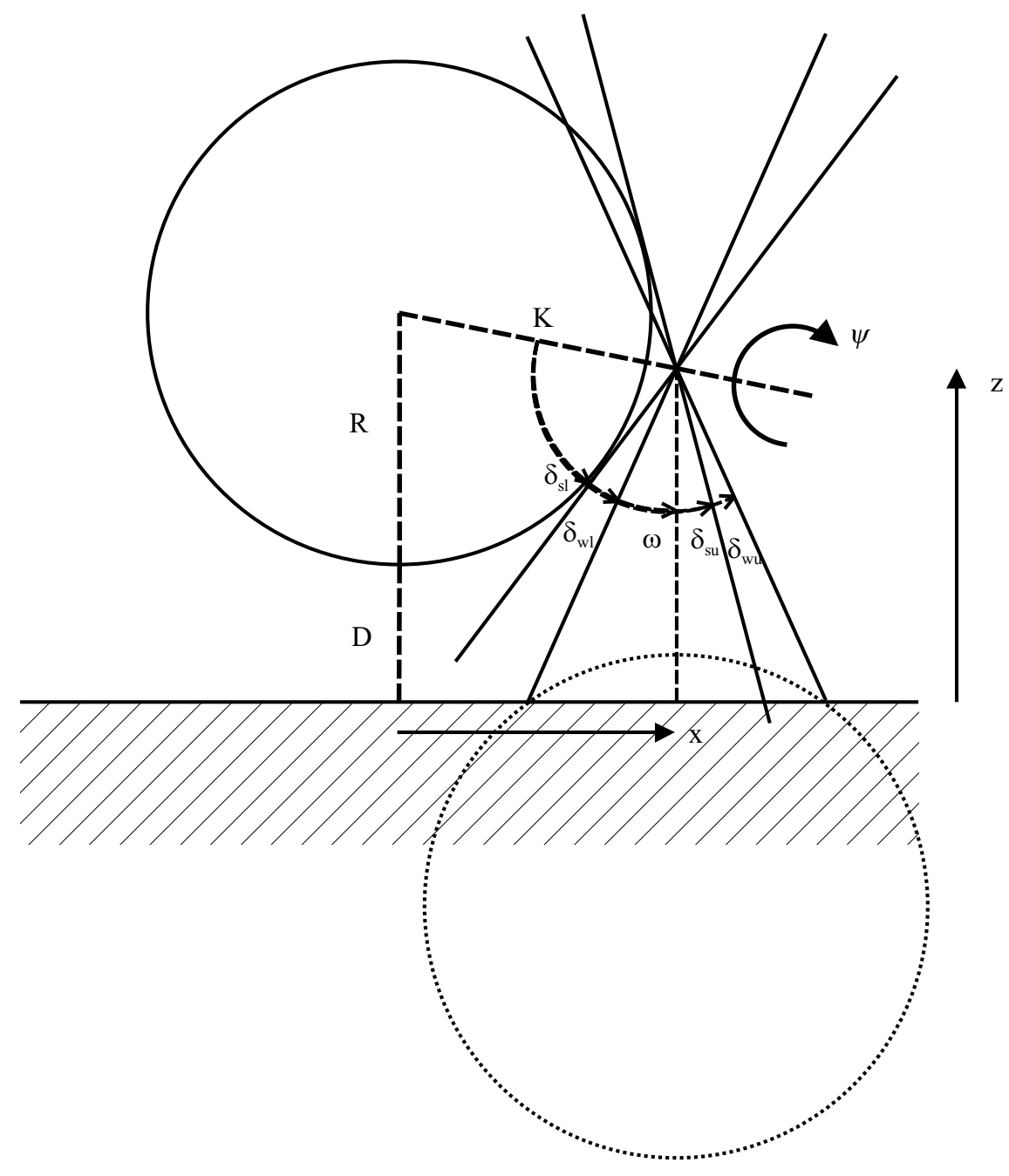

Figure 1 


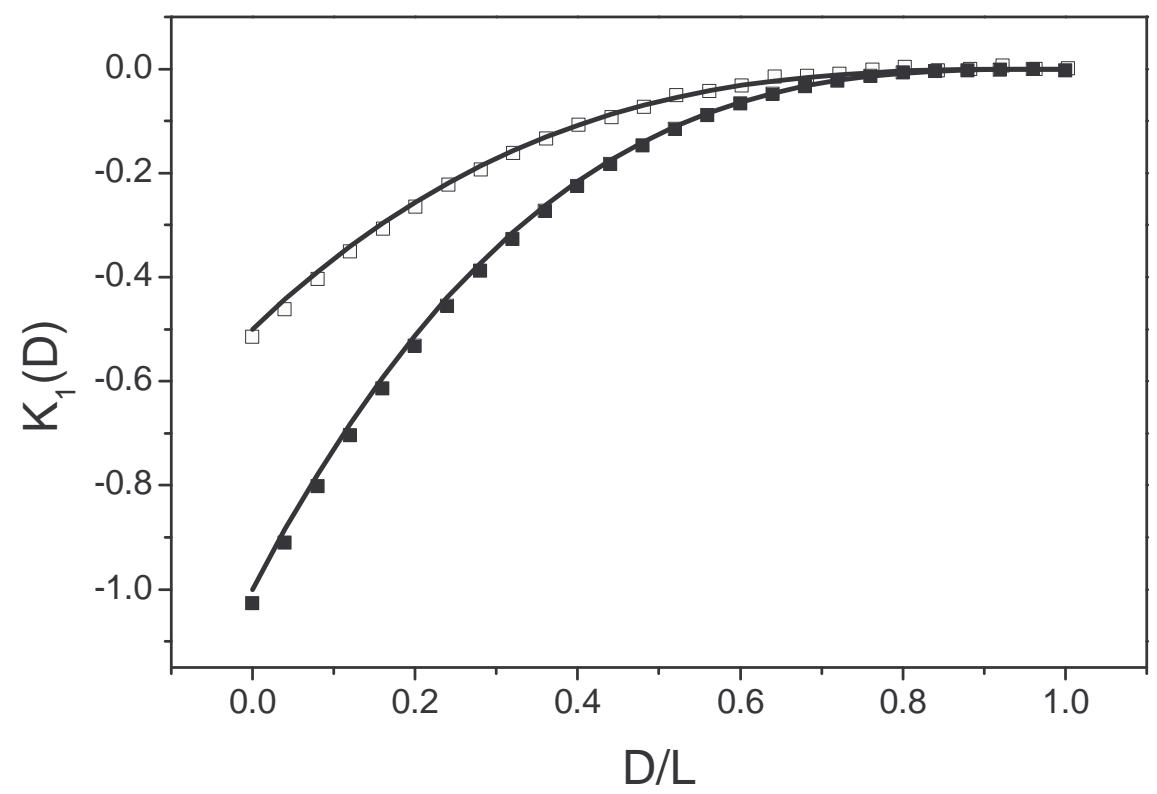

Figure 2 


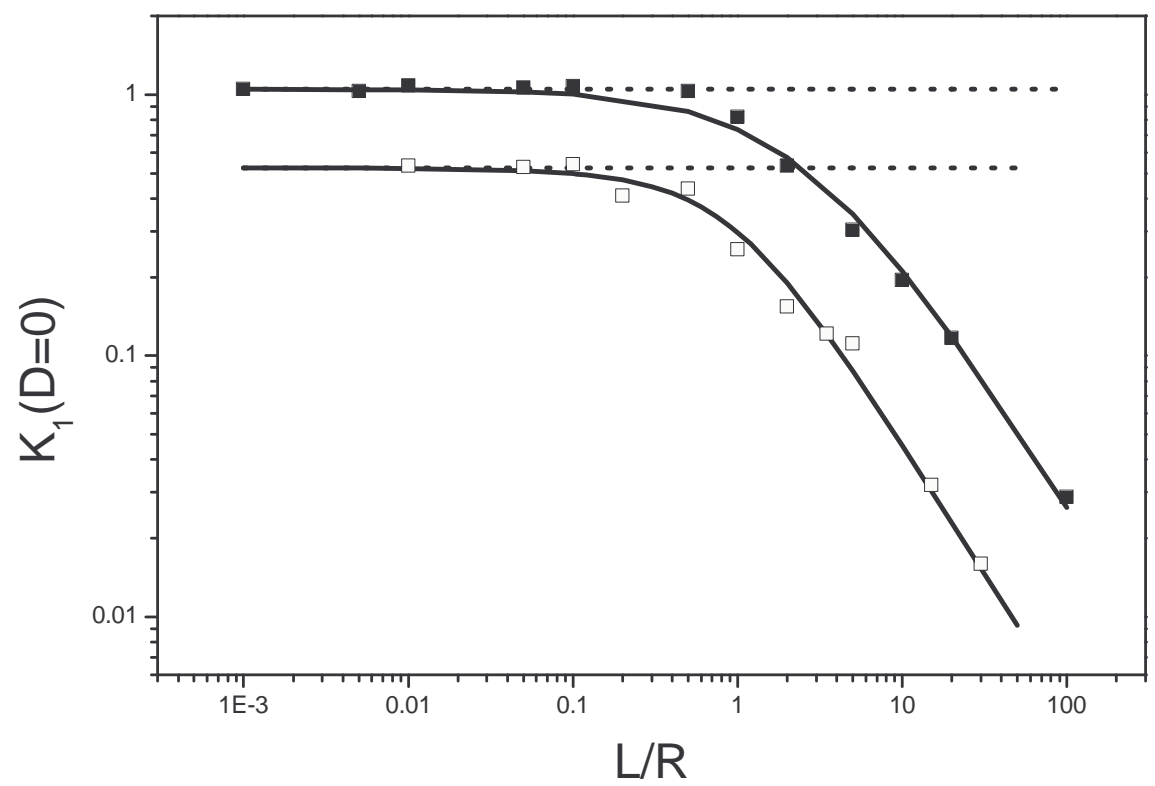

Figure 3 


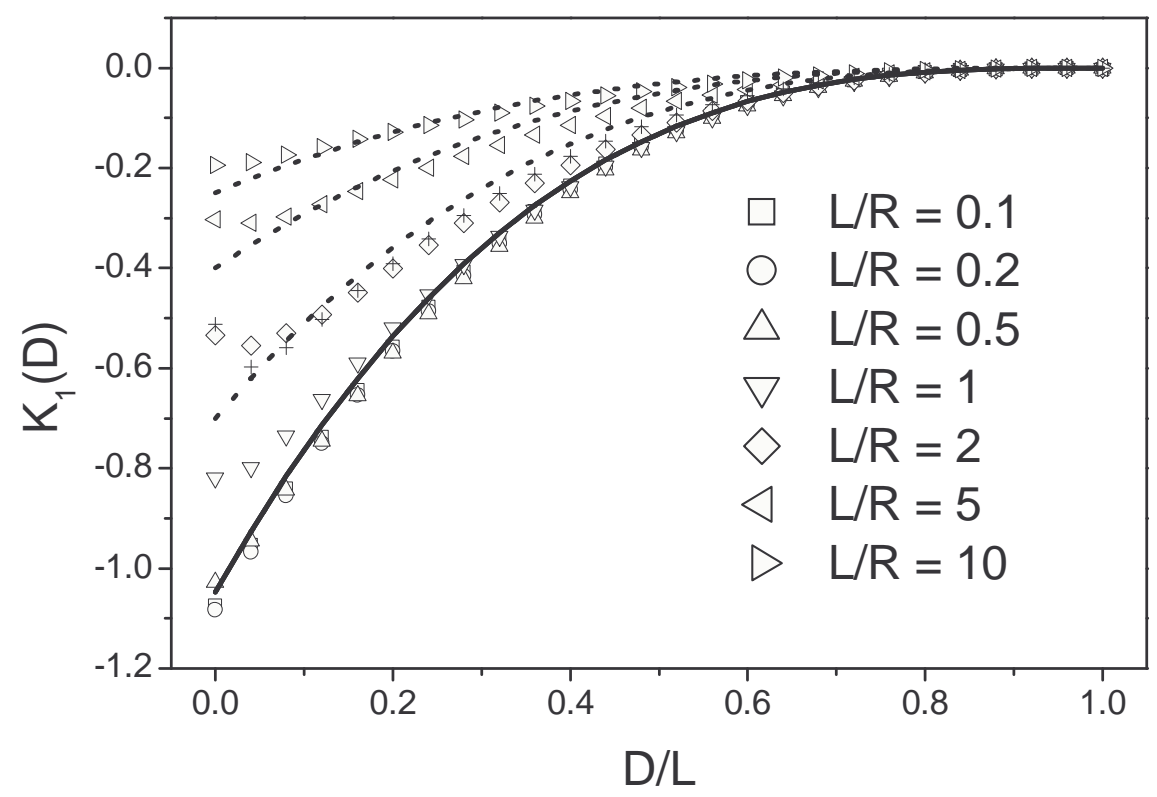

Figure 4 

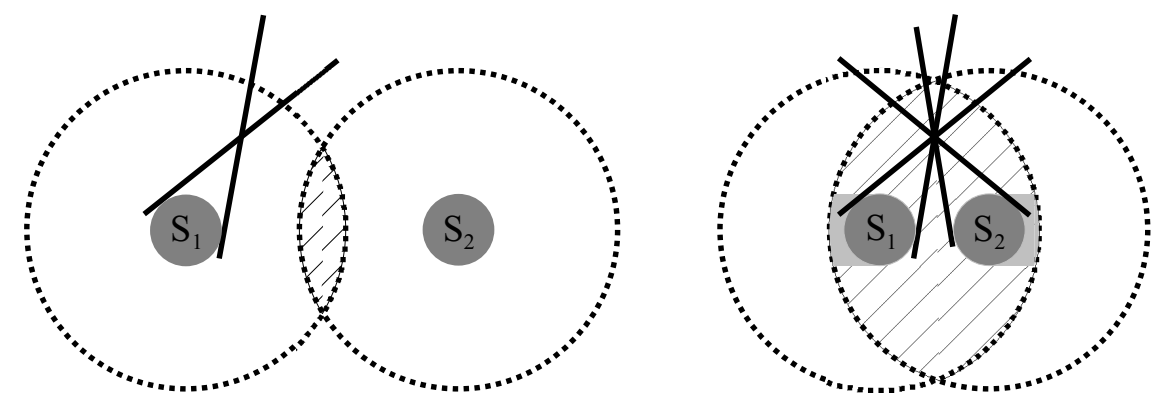

Figure 5 


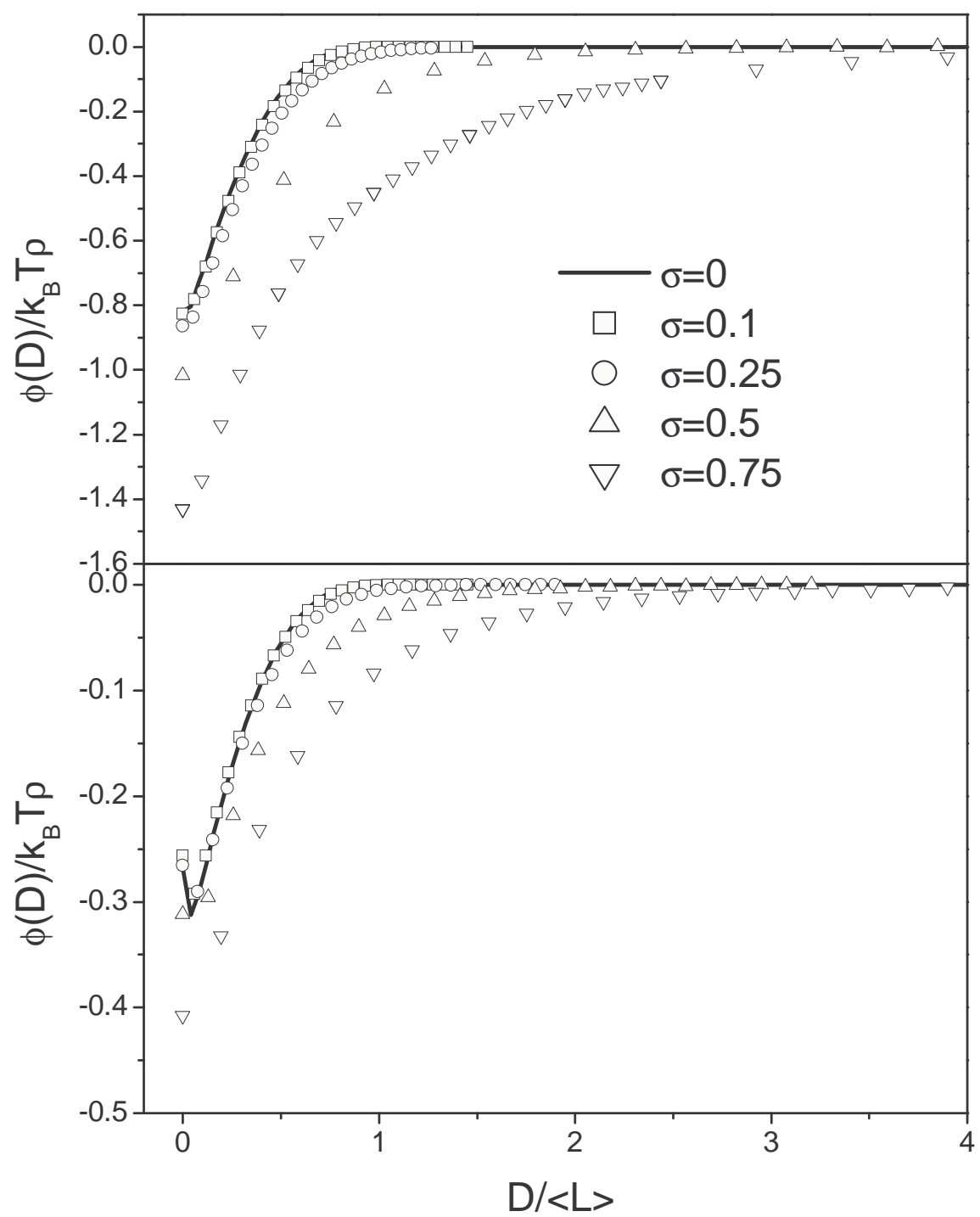

Figure 6 


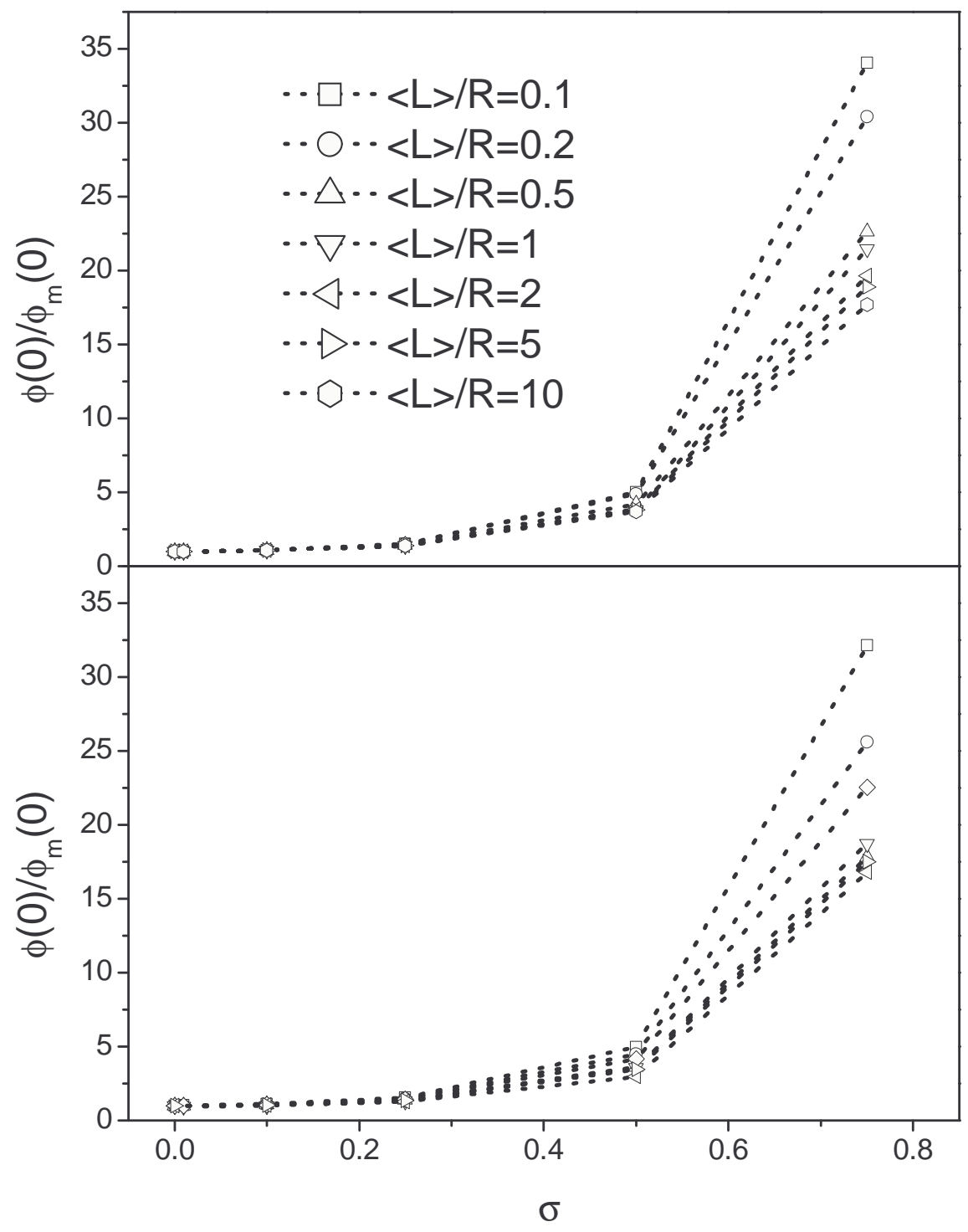

Figure 7 


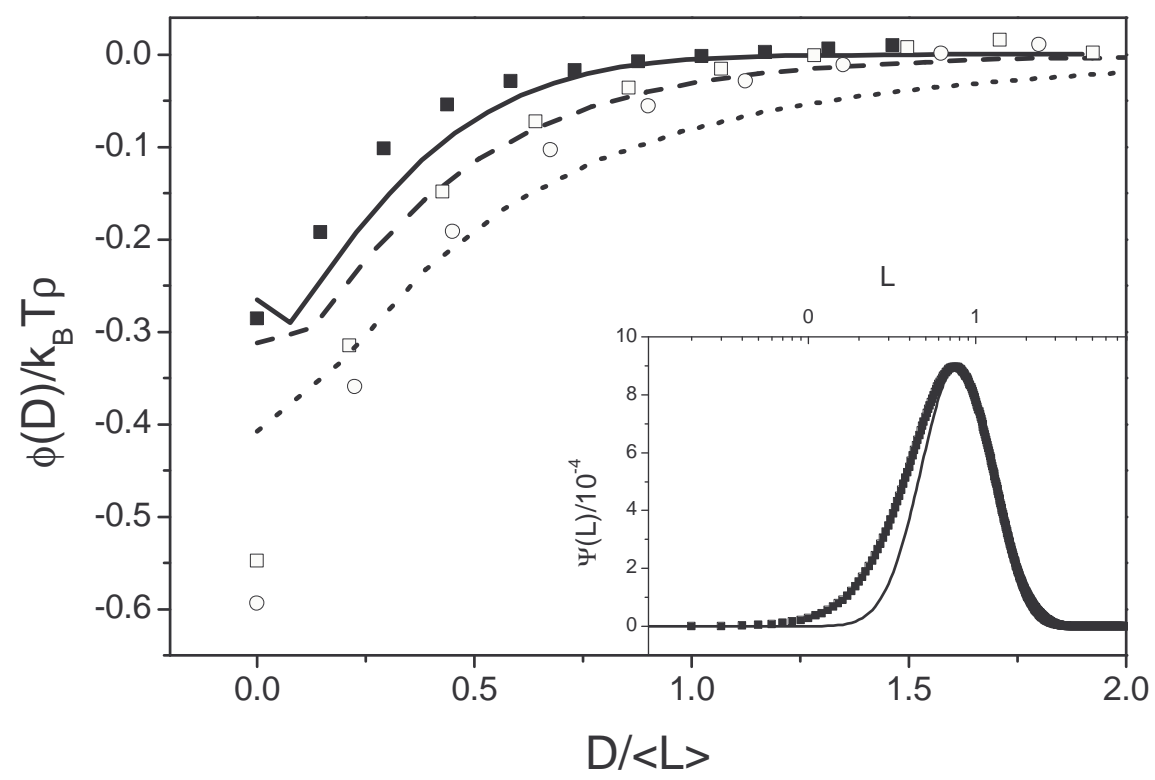

Figure 8 


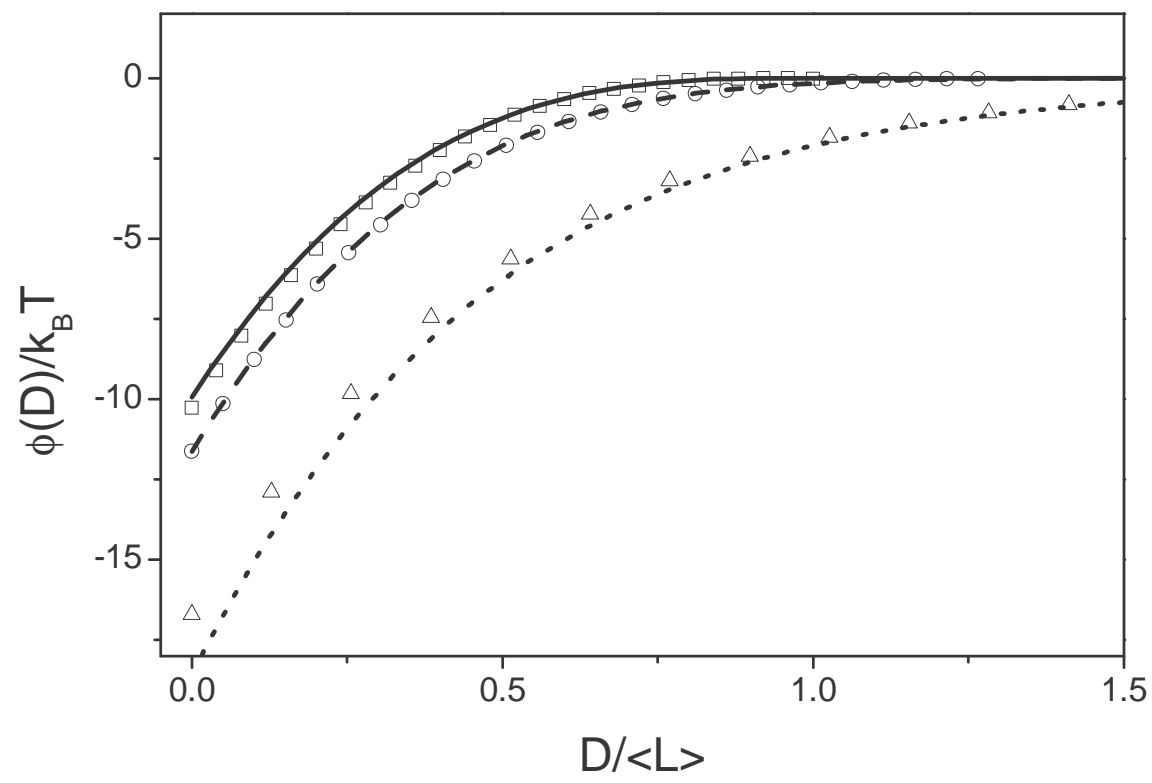

Figure 9 


\section{Captions to Figures}

Figure 1 Basic construction for the calculation of the volume excluded, $V_{e x}(D)$, for the

rod by a sphere and a wall. $V_{e x}(D)$ is calculated by numerical integration as a function of the rod's center position and its orientation. The dotted sphere excludes the same volume for the rod as the wall. With this construction it is also possible to calculate $V_{e x}(D)$ from two spheres.

Figure 2 Comparison of numerically exact results for $K_{1}(D)$ as a function of $D / L$ with the prediction from Derjaguin's approximation. Symbols are data obtained from numerical calculation of the excluded volume in the sphere/sphere (open) and the sphere/wall (solid) configuration for $L / R=0.1$. Full lines represent the corresponding Derjaguin approximation.

Figure 3 Dependence of $K_{1}(D=0)$ on $L / R$. Symbols are data obtained from numerical calculations for the sphere/sphere (open) and the sphere/wall (solid) configuration. Full lines represent three parameter fits with eq. 9 and the dotted horizontal line are the predictions by the Derjaguin approximation.

Figure 4 Dependence of $K_{1}(D)$ on $D / L$ in the sphere/wall configuration for different 
$L / R$ as indicated in the legend. The crosses represent the twofold of the potential calculated for $L / R=1$ in the sphere/sphere configuration. The full line represents the Derjaguin approximation and the dotted curves are a rough guide assuming the same $D / L$ dependence with an amplitude which changes with $L / R$.

Figure 5 Illustration of the counter balancing effects of positional and orientational degrees of freedom. Left: the depletion zones of two spheres indicated by the dotted circles overlap, while the orientation of the rod is hindered only by sphere $S_{1}$. Right: The overlap volume, which is related to the hatched area, increases on further approach of the spheres, leading to a decrease of $V_{e x}(D)$. On the other side, the rod experiences an additional restriction of orientational freedom due to sphere $S_{2}$, which increases $V_{e x}(D)$.

Figure 6 Depletion potentials mediated by polydisperse rods with $\langle L\rangle / R=1$ vs. normalized separation distance for sphere/wall (top) and sphere/sphere (bottom) configuration. Symbols refer to different values of the standard deviation as indicated in the legend. The full lines represent the case of monodisperse rods.

Figure 7 Contact potentials normalized to the value for monodisperse rods as a function of relative standard deviation for sphere/wall (top) and sphere/sphere (bottom) configuration. Symbols are calculated for different $\langle L\rangle / R$ as indicated in the legend and dotted lines are guides to the eye. 
Figure 8 Depletion potentials mediated by polydisperse rods. The lines represent potentials mediated by $\log -$ norm distributed $(\sigma=0.25$ : full, $\sigma=0.5$ : dotted and $\sigma=0.75$ : dashed) ensembles of rods, while the symbols correspond to Schulz-Zimm distributions with $\sigma=0.25$ : full squares, $\sigma=0.5$ : open squares and $\sigma=0.75$ : open circles. Inset: $\log -$ norm (full line) and Schulz-Zimm (symbols) distribution function with $\sigma=0.5$.

Figure 9 Depletion potentials mediated by an ensemble of rods with varying width of the length log-normal distribution. The symbols are exact potentials calculated for constant rod number density, $\rho=955 \mu \mathrm{m}^{-3}$, with $\sigma=0$ (squares), $\sigma=0.25$ (circles) and $\sigma=0.5$ (triangles). The lines represent Derjaguin approximations at constant mass concentration, $c=7.455 \mathrm{~g} / \mathrm{L}$ with with $\sigma=0$ (full), $\sigma=0.25$ (dotted) and $\sigma=0.5$ (dashed). 